\title{
A NOTE ON OPEN MAPS
}

\section{STEPHEN SMALE}

In [1] E. E. Floyd proved that if $X$ and $Y$ are Peano continua and $f: X \rightarrow Y$ is light, open, and onto, then the covering homotopy property for a point holds. Here we will prove (Theorem 1) that under different (roughly speaking, more general) conditions the covering homotopy property for a point holds up to a homotopy. This has certain implications on the induced homomorphism of the fundamental groups (Theorem 2).

1. Definitions. We consider a triple $(X, p, Y)$ to consist of two topological spaces $X$ and $Y$ and a map $p$ from $X$ into $Y$. We denote by $I$ the closed unit interval.

A triple $(X, p, Y)$ has the covering homotopy property for a point if given a map $f: I \rightarrow Y$ and a point $q \in p^{-1} f(0)$, there exists a map $\bar{f}: I \rightarrow X$ with $\bar{f}(0)=q$ and $p \bar{f}=f$. The covering homotopy property for a point holds up to homotopy if given $(X, p, Y), f$ and $q$ as above, there exists a map $\bar{f}: I \rightarrow X$ with $\bar{f}(0)=q, p \bar{f}(1)=f(1)$ and $p \bar{f}$ is homotopic to $f$ with the homotopy fixed on the end points of $I$.

A map is called proper if the inverse images of compact sets are compact. If $A$ is a subset of a space $X$, then $\pi_{1}(A / X)$ will denote the image of $\pi_{1}(A)$ in $\pi_{1}(X)$ under the homomorphism induced by inclusion. A space $S$ will be called semilocally 1-connected if for each point $x \in S$, there exists a neighborhood $U$ of $x$ such that $\pi_{1}(U / S)=1$.

A triple $(X, p, Y)$ will be said to have Property $\mathrm{A}$ if the following is true:

Property A. The space $X$ is locally arcwise connected and Hausdorff; $Y$ is semilocally 1 -connected and metric. The map $p$ is open, proper, and onto.

The main theorem of this note is the following.

Theorem 1. A triple ( $X, p, Y)$ having Property A has the covering homotopy property for a point up to homotopy.

\section{Proof of Theorem 1.}

Lemma 1. Let $Y$ be a metric space and $f: X \rightarrow Y$ be proper and onto. Suppose $y \in Y$ and $U$ is an open set of $X$ containing $f^{-1}(y)$. Then there exists a neighborhood $V$ of $y$ such that $f^{-1}(V) \subset U$.

Presented to the Society, April 14, 1956 under the title The effect of an open map on the fundamental group; received by the editors May 1, 1.956. 
Proof. Suppose the lemma is not true. For each integer $i>0$, let $V_{i}$ be a neighborhood of $y$ of diameter $1 / i$. Then for each $i$ there exists a point $y_{i} \in V_{i}$ with $f^{-1}\left(y_{i}\right) \cap(X-U) \neq \varnothing$. Choose $x_{i} \in f^{-1}\left(y_{i}\right) \cap(X-U)$ for each $i$. The set $K=\cup_{y_{i}} \cup_{y}$ is compact hence $f^{-1}(K)$ is. Therefore the set $\left\{x_{i}\right\}$ has a limit point, say $x$. By the continuity of $f, x \in f^{-1}(y)$. Since $X-U$ is closed, $x \in X-U$, contradicting the previous statement. q.e.d.

REMARK. R. L. Wilder has pointed out to me that if instead of $Y$ being metric, $Y$ is Hausdorff and has a countable basis of neighborhoods at each point then the proof for this lemma is still valid. In all the theorems in this paper, metric may be replaced by possession of this property.

The proof of Theorem 1 proceeds as follows.

For each $y \in Y$ choose by the semilocally 1 -connectedness property of $Y$ a neighborhood $U_{y}$ of $y$ such that $\pi_{1}\left(U_{y} / X\right)=1$. Then for each $x \in p^{-1}(y)$ let $P_{y}(x)$ be an arcwise connected neighborhood of $x$ such that $p\left[P_{y}(x)\right] \subset U_{y}$. By the compactness of $p^{-1}(y)$ choose a finite number of these neighborhoods, say $P_{1 y}, \cdots, P_{k y}$, covering $p^{-1}(y)$. Let $P_{y}=\mathrm{U} P_{i y}$ and $\bar{V}_{y}=\bigcap_{p}\left(P_{i y}\right)$. Since $p$ is open $\bar{V}_{y}$ is an open set of $Y$ containing $y$. Choose by Lemma 1 a neighborhood $V_{y}$ of $y$ contained in $\bar{V}_{y}$ such that $p^{-1}\left(V_{y}\right) \subset P_{y}$.

Let $g: I \rightarrow Y$ be given with $g(0)=y_{0}$ and $x_{0} \in p^{-1}\left(y_{0}\right)$. We will construct a map $\bar{g}: I \rightarrow X$ such that $\bar{g}(0)=x_{0}, p \bar{g}(1)=g(1)$ and $p \bar{g}$ is homotopic to $g$ with fixed endpoints.

Let $\delta$ be the Lebesgue number of the covering of $I,\left\{g^{-1}\left(V_{y}\right) / y \in Y\right\}$. If $I_{0}=\{t \in I / 0 \leq t \leq \delta\}, g\left(I_{0}\right)$ is contained in one of the $V_{y}$ 's say $V$ with $V \subset \bar{V} \subset U, \bar{V}=\bigcap_{p}\left(P_{i}\right), P=\bigcup P_{i}$, and $p^{-1}(V) \subset P$. The open sets here are to correspond in the obvious fashion to those of the previous paragraphs. Then $x_{0}$ will lie in some $P_{i}$ say $P_{1}$. Let $x_{1}$ be an arbitrary point in the nonempty intersection $P_{1} \cap p^{-1}[g(\delta)]$. For $0 \leq t \leq \delta$ define $\bar{g}(t)$ to be an arc in $P_{1}$ with $\bar{g}(0)=x_{0}$ and $\bar{g}(\delta)=x_{1}$. Then by the choice of $U$, for $0 \leq t \leq \delta, g(t)$ is homotopic to $p \bar{g}(t)$ with the homotopy fixed on $t=0$ and $t=\delta$. Iteration will yield a satisfactory definition of $\bar{g}(t)$ for all $t \in I$. This proves Theorem 1 .

REMARK. Examples show that Theorem 1 is false if either of the local connectedness conditions is dropped. Also the qualification "up to homotopy" is necessary.

\section{Applications.}

Corollary 1. If $(X, p, Y)$ is a triple possessing Property A, $y \in Y$, and $x \in p^{-1}(y)$, then the induced transformation $p \neq: \pi_{1}\left(X, p^{-1}(y), x\right)$ $\rightarrow \pi_{1}(Y, y)$ is onto.

This is an immediate consequence of Theorem 1. 
CoRollary 2. Suppose under the conditions of Corollary 1 the single inverse image $p^{-1}(y)$ is arcwise connected. Then the induced homomorphism p $\mathrm{t} \pi_{1}(X, x) \rightarrow \pi_{1}(Y, y)$ is onto.

Corollary 2 follows from Corollary 1 and the exact homotopy" sequence of the pair $\left(X, p^{-1}(y)\right)$.

Theorem 2. Let $(X, p, Y)$ be a triple having Property A. Then the quotient $\pi_{1}(Y, y) / p_{t}\left[\pi_{1}(X, x)\right]$ is finite.

M. L. Curtis [2, p. 239] proved a somewhat weaker theorem using the previously mentioned result of E. E. Floyd. The proof in his paper applys directly here to yield Theorem 2 from Theorem 1 . It will not be repeated.

Theorem 2 is roughly a generalization of a result obtained by $T$. Ganea [3, p. 195]. Ganea proved that under local connectedness conditions, given an open map of a compact Hausdorff space $X$ onto a Hausdorff space $Y$, if $\pi_{1}(X)$ is finite then $\pi_{1}(Y)$ is. Ganea uses Chevalley's definition of fundamental group.

In the following, the homology is the singular theory, $Q$ denotes the rational group and $Z$ the group of integers.

Theorem 3. If $(X, p, Y)$ is a triple possessing Property A and $X$ is arcwise connected then the induced homomorphism $p_{*}: H_{1}(X ; Q)$ $\rightarrow H_{1}(Y ; Q)$ is onto.

Proof. The Hurewicz homomorphism $h: \pi_{1}(Y) \rightarrow H_{1}(Y)$ induces a homomorphism $h_{*}: \pi_{1}(Y) / p_{\sharp}\left[\pi_{1}(X)\right] \rightarrow H_{1}(Y ; Z) / p_{*}\left[H_{1}(X ; Z)\right]$. Since $h$ is onto, $h_{t}$ is also onto. Then from Theorem 2 it follows that $H_{1}(Y ; Z) / p_{*}\left[H_{1}(X ; Z)\right]$ is finite. Theorem 3 follows from the universal coefficient theorem for homology.

It might be mentioned that Whyburn [4] proved a result similar to Theorem 3 using rational Vietoris homology. The only differences are that his spaces were compact metric without local connectedness conditions and he assumed that the Vietoris group $H_{1}(X ; Q)$ was finitely generated.

\section{BIBLIOGRAPHY}

1. E. E. Floyd, Some characterizations of interior maps, Ann. of Math. vol. 51 (1950) pp. 571-575.

2. M. L. Curtis, Deformation free continua, Ann. of Math. vol. 57 (1953) pp. 231247.

3. Tudor Ganea, Simply-connected spaces, Fund. Math. vol. 38 (1951) pp. 179-203.

4. G. T. Whyburn, The mapping of Betti groups under interior transformations, Duke Math. J. vol. 4 (1938) pp. 1-8.

UNIVERSITY OF MiCHIGAN 